\title{
Identification of an Aminoacridine Derivative That Binds to RNA Tetraloops
}

\author{
Zhaohui Yan, ${ }^{\dagger}$ Shweta Sikri, ${ }^{\dagger}$ David L. Beveridge, ${ }^{\dagger}$ and Anne M. Baranger*,†, \\ Department of Chemistry and Molecular Biophysics Program, Wesleyan University, Middletown, Connecticut 06459, and Department of \\ Chemistry, 361 Roger Adams Laboratory, University of Illinois, 600 S. Mathews Avenue, Urbana, Illinois 61801
}

Received March 16, 2007

RNA folds into diverse structures that form unique targets for small molecules and thus provide significant potential for controlling biological processes involving RNA with small-molecule ligands. We are investigating molecular recognition of tetraloop RNA by small molecules. RNA tetraloops are four-nucleotide stem loops with unusual stability that are involved in biological processes involving RNA by forming binding sites for proteins and other RNAs. We have sequentially used the docking programs DOCK and AutoDock to screen 1990 small molecules in the NCI diversity set to identify molecules selective for RNA tetraloops over double-stranded RNA. The compounds predicted to bind to tetraloop RNA were evaluated for binding RNA tetraloops using ${ }^{1} \mathrm{H}$ NMR spectroscopy and fluorescence techniques. An aminoacridine derivative (AD2) was identified that binds to a GAAA tetraloop in a $2: 1$ ratio with dissociation constants of 1.0 and $4.0 \mu \mathrm{M}$. AD2 binds with approximately 20-fold and 9-fold higher affinity to tetraloop RNA than to double- and single-stranded RNAs, respectively.

\section{Introduction}

RNA plays essential and multifaceted roles in gene expression. For example, RNA is the information carrier between DNA and proteins, a component of the machineries that perform RNA processing steps and translation, and an essential regulator of many steps in gene expression. ${ }^{1}$ Therefore, small molecules that could bind to RNA and affect these biological functions would be of great utility. ${ }^{2-5}$ Small molecules that bind to bacterial ribosomal RNA and inhibit protein synthesis are used as antibiotics. ${ }^{6,7}$ However, there is considerable potential for small molecules to target other processes involving RNA. RNAs fold into complex structures that form diverse binding sites for small molecules. ${ }^{8}$ RNA motifs that are the target sites for proteins and other RNAs and are important for the correct functioning of the RNA form attractive targets for small molecules. We report here investigations of small-molecule recognition of RNA hairpins, in particular a common, small RNA hairpin called the GNRA tetraloop.

GNRA tetraloops are exceptionally stable stem loops that were first identified from sequence comparisons of ribosomal RNAs. $^{9-11}$ More than $55 \%$ of loops in the $16 \mathrm{~S}$ rRNA are tetraloops, and most of these have the consensus sequence GNRA. ${ }^{10,12}$ GNRA tetraloops are known to be involved in functionally important tertiary contacts in catalytic RNA, ${ }^{13-16}$ ribosomal RNA, ${ }^{17,18}$ and the viral internal ribosome entry site (viral IRES) $)^{19-21}$ and are also known to interact with proteins such as SRP proteins, ribosomal proteins, elongation factors, and sarcin and ricin toxins. ${ }^{11,18,22-26}$ GNRA tetraloops have been extensively studied by NMR, ${ }^{27-31}$ X-ray crystallography, ${ }^{32-37}$ chemical modification, ${ }^{38,39}$ thermal melting, ${ }^{11,40}$ Raman and fluorescence spectroscopies, ${ }^{41-44}$ and molecular dynamics simulations. ${ }^{45-47}$ Compared with the extensive structural and thermodynamic studies on RNA tetraloops, there is surprisingly little known about small-molecule recognition of RNA tetraloops. A solution structure of a GAAA tetraloop complexed with cobalt(III) hexaamine revealed that the metal ion interacts

* To whom correspondence should be addressed. Phone: (217) 2447649. Fax: (217) 244-8024. E-mail: baranger@uiuc.edu.

Wesleyan University.

$\doteqdot$ University of Illinois
A.

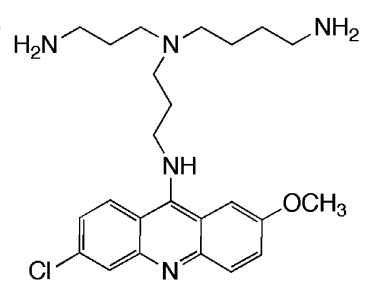

B.

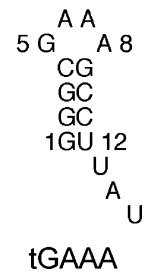

Figure 1. (A) Aminoacridine derivative (AD1) previously found to bind RNA tetraloops. ${ }^{54}$ (B) Sequence of tGAAA RNA tetraloop used in binding experiments with $\mathrm{AD} 1$.

with the RNA tetraloop in the major groove. ${ }^{30}$ Deoxystreptamine dimers have recently been shown to bind to GAAA and UUCG tetraloop RNAs with submicromolar affinity and with selectivity for tetraloops over stem loops with different loop sizes. ${ }^{48,49}$ Recognition of hairpins in general by small molecules is rare. ${ }^{50-53}$ Thus, GNRA tetraloops comprise a structurally characterized and biologically important class of RNA hairpin that has been only minimally explored as a target for smallmolecule ligands.

We have previously characterized the binding of an aminoacridine derivative (AD1) to a GAAA RNA tetraloop (tGAAA, Figure 1) ${ }^{54} \mathrm{AD} 1$ was initially identified as an inhibitor of the Tat-TAR interaction with an $\mathrm{IC}_{50}$ of $22 \mathrm{nM} .^{55,56} \mathrm{We}$ found that AD1 forms a 1:1 complex with the tGAAA tetraloop and binds to the junction between the loop and the stem of the tGAAA tetraloop with a $K_{\mathrm{D}}$ of $1.6 \mu \mathrm{M}$. However, AD1 does not bind specifically to the tGAAA tetraloop. For example, the binding affinities of AD1 for tetraloop and duplex RNAs were found to be equivalent. ${ }^{57}$ Therefore, we initiated a computational screen to identify molecules, not necessarily related to AD1, that would be able to bind tetraloop RNA with improved selectivity for tetraloop over duplex RNA.

Computational docking is a common tool used toward the identification of small-molecule ligands of macromolecules. ${ }^{58,59}$ However, compared to proteins, RNA is a relatively new target for computational docking. Most docking programs have been designed for proteins, and only a few have been used to identify small-molecule ligands for RNA targets. ${ }^{7,60-72}$ Two programs that have been evaluated for their ability to reproduce experi- 
Table 1. Computational and Experimental Binding Free Energies of the Test Set of RNA-Small-Molecule Complexes

\begin{tabular}{|c|c|c|c|c|c|}
\hline \multirow[b]{2}{*}{ ligand-RNA complex } & \multicolumn{2}{|c|}{ DOCK } & \multicolumn{2}{|c|}{ AutoDock } & \multirow{2}{*}{$\begin{array}{c}\text { experiment } \\
\Delta G(\mathrm{kcal} / \mathrm{mol})\end{array}$} \\
\hline & $\overline{\text { energy }\left(\mathrm{DU}^{a}\right)}$ & $\operatorname{rmsd}(\AA)$ & $\overline{\text { energy }(\mathrm{kcal} / \mathrm{mol})}$ & $\operatorname{rmsd}(\AA)$ & \\
\hline arginine-RNA aptamer $^{b}$ & -19 & 3.5 & -6.71 & 1.87 & $-5.76^{c}$ \\
\hline biotin-RNA pseudoknot ${ }^{b}$ & -25 & 1.56 & -8.59 & 1.19 & $-7.12^{c}$ \\
\hline theophylline--RNA ${ }^{b}$ & -34.5 & 0.8 & -8.55 & 1.35 & $-8.85^{c}$ \\
\hline
\end{tabular}

${ }^{a}$ DU is dock unit. ${ }^{b}$ The PDB codes are $1 \mathrm{KOC}$ for the arginine-RNA aptamer complex, ${ }^{76} 1 \mathrm{~F} 27$ for the biotin-RNA pseudoknot complex, ${ }^{77}$ and $1 \mathrm{EHT}$ for the theophylline-RNA aptamer complex. ${ }^{78}{ }^{c}$ The experimental binding energies were reported previously for the arginine-RNA aptamer, ${ }^{92}$ the biotinRNA pseudoknot, ${ }^{93}$ and the theophylline-RNA aptamer ${ }^{94}$ complexes.

mental data ${ }^{60,63,68-70,72}$ and used to find small-molecule ligands for RNA ${ }^{64-67}$ are DOCK and AutoDock. DOCK uses shape complementarity to match the ligand to the binding site of the receptor. ${ }^{73}$ The accuracy of DOCK is limited by its scoring function, which only considers van der Waals and electrostatic interactions. AutoDock uses a Lamarckian genetic search algorithm and a scoring function that includes desolvation, hydrogen bonding, ligand torsional, van der Waals, and electrostatic energies. ${ }^{74}$ The ability of these programs to reliably predict the binding sites and relative affinities of known ligands has been investigated in both RNA and DNA systems. ${ }^{60,70}$ These investigations have suggested that AutoDock is more effective than DOCK but that sequential docking with DOCK, followed by AutoDock, is a better procedure than using either program alone for identifying small molecules in a library that bind the RNA target. However, it is important to note that computational docking to RNA targets is still at an early stage of development, and a recent critical evaluation of 10 docking programs against protein targets, including DOCK but not AutoDock, did not identify any one program as being superior to the others. ${ }^{75}$

We performed sequential docking with DOCK and AutoDock to identify molecules in the diversity set database from the National Cancer Institute able to bind to RNA tetraloops with specificity over duplex RNA. We targeted molecules to a binding pocket in the major groove of the helix proximal to the loop. This site was predicted to provide contacts to support high binding affinity within a structure sufficiently different from duplex RNA to enable selective recognition. These investigations have revealed an aminoacridine derivative (AD2) that is related to AD1 and binds to RNA hairpins with approximately 10-fold and 20-fold selectivity over single-stranded and doublestranded RNA sequences, respectively.

\section{Results and Discussion}

Computational Screening of a Trial Set of RNA-Ligand Complexes. We selected three known RNA-ligand complexes as a small test set for the DOCK and AutoDock programs, an arginine-RNA aptamer complex, ${ }^{76}$ a biotin-RNA pseudoknot complex, ${ }^{77}$ and a theophylline-RNA complex. ${ }^{78}$ These three ligands represent neutral, positively, and negatively charged ligands. Table 1 shows the results of docking of the test set. DOCK ranked these three compounds in the same order as that of the experimental binding energies and was able to dock the biotin and theophylline ligands back to their original positions. However, the complex formed with arginine had a root-meansquared deviation (rmsd) of $3.5 \AA$ from the experimentally determined structure perhaps because of the nonexhaustive sampling and lack of a solvation term in the DOCK scoring function, as discussed previously by James and co-workers. ${ }^{79}$ In contrast, AutoDock docked each ligand to the RNA with an rmsd within $2 \AA$ of the experimentally determined structure. The free energies of binding calculated by AutoDock approach the values determined experimentally with an error of less than $1.5 \mathrm{kcal} / \mathrm{mol}$ in all three test complexes. These results agree with those obtained by Varani and co-workers in a detailed

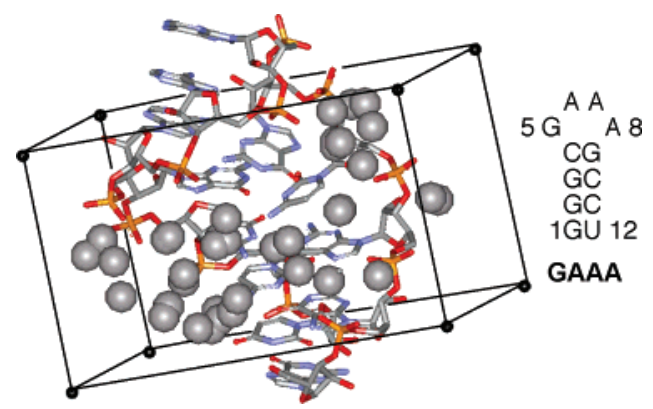

Figure 2. Docking site of 38 spheres generated by DOCK on the GAAA tetraloop. The sizes of the spheres are arbitrary and were chosen for ease of visualization. The edges of the energy scoring grid that was constructed based on these spheres are shown.

analysis of the DOCK and AutoDock programs for RNA-ligand complexes. ${ }^{60}$

Computational Docking of the NCI Diversity Set to a GAAA RNA Tetraloop. We screened the NCI diversity set of compounds for molecules that bind RNA tetraloops. This database is composed of 1990 compounds that are derived from 140000 compounds at the National Cancer Institute (NCI). We chose this database because it is relatively small, it contains a diverse set of compounds that are known to be druglike, and selected compounds can be easily obtained from the NCI. We chose to use DOCK to eliminate about $80 \%$ of the database and then use AutoDock to calculate the binding free energy of the remaining compounds.

A structure of the tGAAA RNA tetraloop determined by Pardi and co-workers was used as the target for the computational docking. ${ }^{80,81}$ This 15 mer RNA has a $3^{\prime}$-overhang that was too flexible to define by NMR. Thus, the nucleotides in the $3^{\prime}-$ overhang were not included in the docking calculations.

As preparation for performing computational docking, a binding site for the small-molecule libraries on the GAAA RNA tetraloop was defined for DOCK using a model of the AD1GAAA tetraloop complex determined by AutoDock. AutoDock scanned the entire RNA molecule to find a site at which the small molecule interacted with the RNA with the lowest free energy of binding. AD1 was found to bind to the major groove of the GAAA RNA tetraloop in the majority of the predicted stable structures, including the top-scoring structure. This binding site is consistent with the results of our previously reported footprinting experiments of the AD1-tGAAA tetraloop complex, which indicated that AD1 binds to the junction between the loop and the stem of the tetraloop. ${ }^{54}$ In addition, this binding site is similar to that determined for cobalt(III) hexammine on a GAAA tetraloop by NMR. ${ }^{82}$

DOCK uses shape complementarity to match the ligand to the binding site of the receptor by generating a sphere file to represent the docking site. The major groove docking site was assigned 38 spheres by DOCK, which were used to create an energy scoring grid. The spheres and the edges of the scoring grid are shown in Figure 2. The spheres are located in the major groove extending into the loop region. This region of the 
$\mathrm{NCl}$ diversity set (1990 compounds)

7 Assign partial charges by INSIGHT II

$\checkmark$ filter by molecular weight $(<500)$

1400 compounds

DOCK to tetraloop RNA

$\checkmark$ select top $50 \%$ binders

700 compounds

DOCK to duplex RNA

select compounds with higher affinty for tetraloop than duplex RNA

350 compounds

$\llbracket$ AutoDock to tetraloop RNA

95 compounds

$\sqrt{ }$ Evaluate binding experimentally

Figure 3. Flow chart of computational screening of the NCI diversity set with DOCK and AutoDock programs.

tetraloop forms a pocket for binding with small molecules and includes loop nucleotides, although not all of the loop bases. Thus, we expected that the identification of small molecules that bind RNA tetraloops with selectivity against nonhairpin structures, such as duplex RNA, would be possible using this binding site.

The computational screening of the NCI diversity set for molecules to bind to the GAAA tetraloop is outlined in Figure 3. Compounds from the NCI diversity set with a molecular weight less than 500 were assigned partial atomic charges using the Insight II CFF91 force field and were docked to the GAAA tetraloop RNA using DOCK. Compounds were ranked by the energy scores determined by DOCK. Half of the database was discarded on the basis of these energy scores. The remaining compounds were subsequently docked to duplex RNA in order to identify ligands that selectively bind to the tetraloop RNA over duplex RNA. The structure of rUAAGGAGGUGAUrAUCACCUCCUUA, which was determined at $2.6 \AA$ resolution by X-ray crystallography, ${ }^{83}$ was chosen for the docking to duplex RNA. The binding site was chosen to be the major groove of base pairs 4-9, as previously described by Kuntz and co-workers. ${ }^{64}$ There were 350 compounds that were predicted to have a higher affinity for tetraloop RNA than for duplex RNA. The program AutoDock was then used to dock these 350 compounds to the tetraloop RNA in order to achieve more accurate energy scores. AutoDock predicted energy scores lower than $-9 \mathrm{kcal} / \mathrm{mol}$ for 95 compounds.<smiles>COc1ccc2nc3cc(Cl)ccc3c(Nc3ccc(O)c(CN4CCN(C)CC4)c3)c2c1</smiles>

NSC130813 (AD2)<smiles>COc1cc(NC(=N)N)c2ncccc2c1</smiles>

Selection of Compounds for Experimental Assays. Of the 95 compounds identified by AutoDock, 29 were selected for experimental investigations based on the diversity of their structural scaffolds. Only 10 of these compounds were soluble in water or in 1:1 or 1:2 water-DMSO solutions. The identities of these 10 compounds were confirmed by ${ }^{1} \mathrm{H}$ NMR spectroscopy. The ${ }^{1} \mathrm{H}$ NMR spectra were found to be inconsistent with the reported structures for 2 of the 10 compounds. The structures of the remaining 8 compounds are shown in Figure 4. Binding to two different GNRA tetraloops, a GAAA and a GCAA tetraloop, was evaluated by NMR, CD, and fluorescence assays, as described below. Both of these tetraloop sequences have been structurally characterized by NMR by Pardi and co-workers. ${ }^{27,28}$ The structures of the two tetraloops are nearly identical except for the position of A6 in the GAAA loop and C6 in the GCAA loop. A6 is stacked on A7 in the GAAA tetraloop, while C6 does not stack on A7 in the GCAA tetraloop. Thus, we expected the selected ligands to bind with similar affinity to both tetraloops.

Screening of Selected Compounds by NMR. Binding to a GCAA tetraloop (tGCAA, Figure 5) was investigated by monitoring changes in the chemical shifts of the RNA as increasing concentrations of individual compounds were added to the solution of the tetraloop RNA. All of the experiments were performed in $50 \mathrm{mM} \mathrm{NaH} \mathrm{PO}_{4} / \mathrm{Na}_{2} \mathrm{HPO}_{4}\left(90 \% \mathrm{H}_{2} \mathrm{O}, 10 \%\right.$ $\mathrm{D}_{2} \mathrm{O}, \mathrm{pH} 7.0$ ) at 5 or $25{ }^{\circ} \mathrm{C}$. Three of the compounds, NSC130813 (AD2), NSC3589 (QD1), and NSC62598, induced changes of the chemical shifts of the aromatic and sugar protons of the RNA upon addition to the RNA. No changes in the imino proton resonances were observed in these titrations. Addition of AD2 induced broadening of the RNA and AD2 signals, which may be due to an exchange rate that is intermediate on the NMR time scale. We investigated the binding of AD2 to tetraloop RNA in detail because the magnitudes of the changes of the chemical shifts of the RNA upon addition of AD2 were larger than those of the RNA upon addition of NSC62598, and the binding affinity of AD2 for RNA is 10- to 100-fold greater than that of QD1 for RNA.

Evaluation of Binding of AD2 to Tetraloop RNA by ${ }^{1} \mathbf{H}$ NMR Spectroscopy. A series of spectra of the aromatic protons of tGCAA upon titration with AD2 are shown in Figure 5. The largest changes in chemical shifts occur in the downfield regions of these spectra. In particular, resonances assigned to A14 and

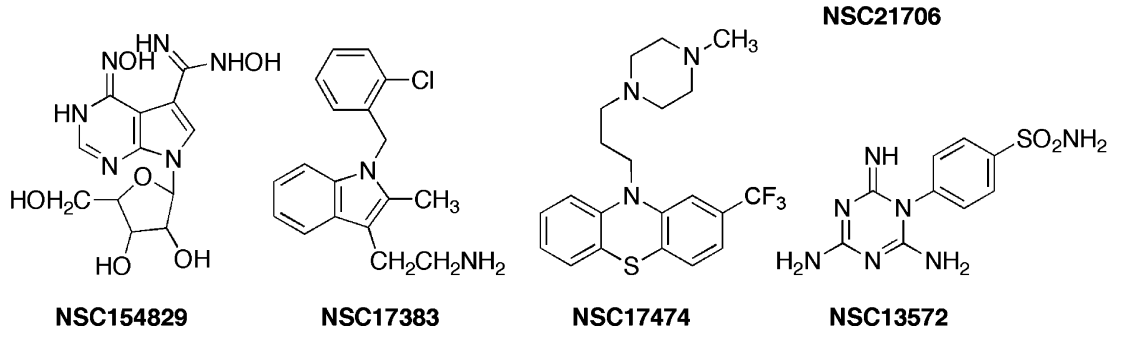

Figure 4. Compounds from computational docking that were studied experimentally. 


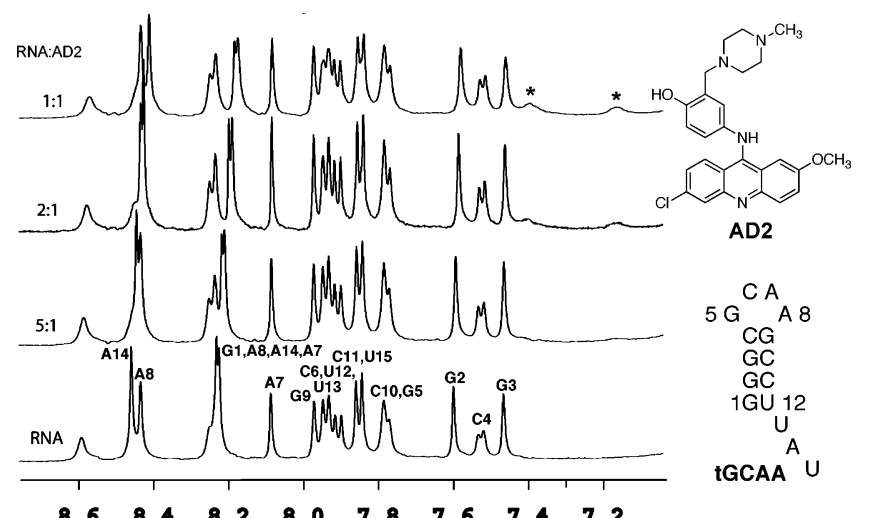

$\begin{array}{llllllll}8.6 & 8.4 & 8.2 & 8.0 & 7.8 & 7.6 & 7.4 & 7.2\end{array}$

Figure 5. Spectra of the aromatic protons of tGCAA upon titration with increasing concentrations of AD2. Experiments were performed at $25^{\circ} \mathrm{C}$ with an RNA concentration of $300 \mu \mathrm{M}$ in a buffer containing $50 \mathrm{mM} \mathrm{NaH}_{2} \mathrm{PO}_{4}, \mathrm{pH} 7.0$, with $90 \% \mathrm{H}_{2} \mathrm{O}$ and $10 \% \mathrm{D}_{2} \mathrm{O}$. Resonances of $\mathrm{AD} 2$ are marked with asterisks.

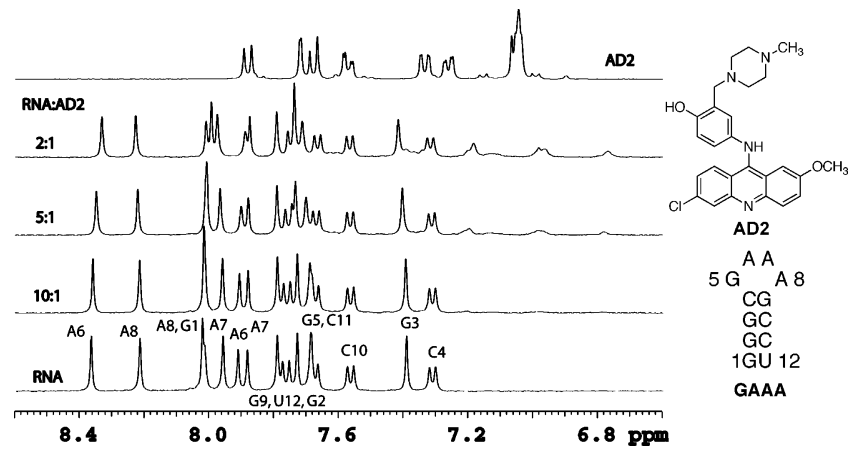

Figure 6. ${ }^{1} \mathrm{H}$ NMR spectra of GAAA tetraloop $(300 \mu \mathrm{M})$ titrated with increasing concentrations of $\mathrm{AD} 2$ in $10 \mathrm{mM} \mathrm{NaH}_{2} \mathrm{PO}_{4}$ (pH 6.0), $\mathrm{D}_{2} \mathrm{O}$ at $40{ }^{\circ} \mathrm{C}$. A spectrum of free $\mathrm{AD} 2$ is shown at the top of the figure.

two sets of overlapping resonances assigned to G1, A8, A14, and A7 for the first set and to C6, U12, and U13 for the second set shift upon addition of AD2. The chemical shifts of the sugar resonances also changed upon addition of AD2, but overlap of these resonances makes evaluation of these data difficult. Because of problems with precipitation of AD2 at higher concentrations, we discontinued the titrations at a 1:1 AD2/ RNA ratio and were unable to observe by NMR the 2:1 complex that is indicated by the fluorescence experiments described below. The observed changes in chemical shift suggest that AD2 is binding to both the base of the loop and the $3^{\prime}$-overhang of tGCAA RNA. The changes in chemical shifts for the overhang resonances are more significant, with the changes in A14 being particularly large. However, assignment of a binding site with this data is ambiguous because binding of AD2 could cause conformational changes remote from the site of binding, and these would also result in chemical shift changes. We were unable to localize the binding site of AD2 using NOE experiments presumably either because of the intermediate rate of exchange of $\mathrm{AD} 2$, which leads to broad $\mathrm{AD} 2$ resonances, or because no protons of AD2 are close enough to protons on the RNA to result in an NOE.

To evaluate the contribution of the 3 '-overhang sequence to the binding of AD2 to tetraloop RNA, the GAAA tetraloop was titrated with AD2. A series of spectra of the aromatic protons of the GAAA tetraloop upon addition of AD2 are shown in Figure 6. Precipitation of AD2 prevented acquisition of spectra at $\mathrm{AD} 2 / \mathrm{GAAA}$ ratios greater than 1:2. The resonances assigned to the loop bases G5, A6, A7 and to the stem bases G1 and

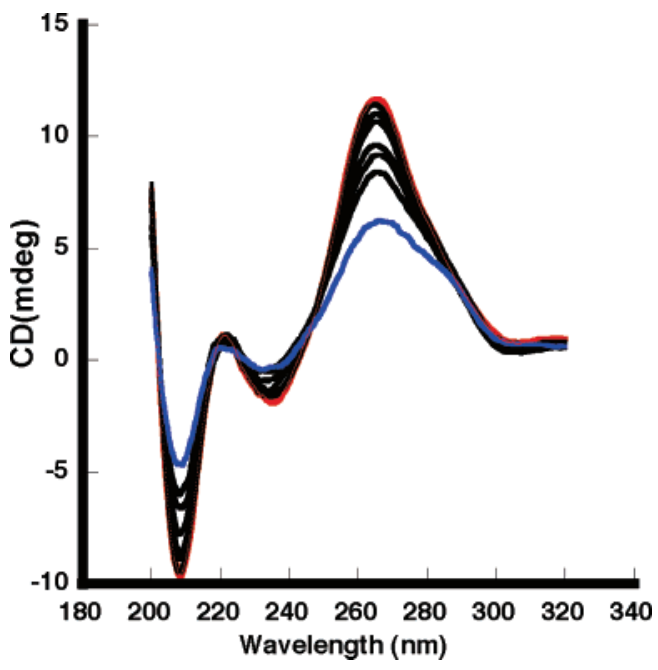

Figure 7. CD spectra of tGCAA $(12 \mu \mathrm{M})$ upon addition of AD2 in $10 \mathrm{mM} \mathrm{NaH} \mathrm{PO}_{4}(\mathrm{pH} 6.0)$ at $25^{\circ} \mathrm{C}$. The concentration of AD2 was varied between 3 and $70 \mu \mathrm{M}$. The spectrum of the free tGCAA RNA is shown in red, and the spectrum with the highest concentration of AD2 $(70 \mu \mathrm{M})$ is shown in blue.

U12 are observed to change upon addition of AD2. The changes in the chemical shifts of the loop residues are larger than was observed for tGCAA described above, suggesting that the AD2 binding site may involve loop nucleotides for the GAAA tetraloop.

Characterization of RNA Tetraloop Structure upon Addition of AD2 by CD Spectroscopy. In order to probe whether the chemical shift changes described above resulted from direct contact of the tGCAA tetraloop with AD2 or from an RNA conformational change upon AD2 binding, we probed changes in RNA conformation upon addition of AD2 by CD spectroscopy. These experiments were performed over a greater range of ratios of $\mathrm{AD} 2$ to RNA than was possible in the NMR experiments because precipitation of AD2 did not occur at the lower overall concentrations required for detection by $\mathrm{CD}$. A decrease in the magnitude of the RNA CD signals at 208 and $265 \mathrm{~nm}$ upon addition of AD2, shown in Figure 7, indicated that the RNA experienced conformational changes upon binding. These results suggest that a portion of the NMR chemical shift changes observed upon addition of AD2 to tetraloop RNA may result from conformational changes in the RNA.

Binding Affinity of AD2 for Tetraloop RNA. The equilibrium binding constants of the AD2-tetraloop RNA complexes were measured by fluorescence techniques using tetraloop RNA labeled with 2-aminopurine (Figure 8). Two labeled tetraloops were used in these experiments: a tGAAA tetraloop labeled with 2-aminopurine at position 8 in the loop (tGAAAp) and a tGCAA tetraloop labeled with 2-aminopurine in the 3'-overhang sequence (tGCAA-UApU). Because the amino groups of both adenine and 2-aminopurine are in the minor groove of the RNA, we expected that the 2-aminopurine substitution would not affect binding of AD2 to the tetraloop RNA. UV melting experiments performed previously showed that the 2-aminopurine substitution for A8 in the loop did not change the stability of the tetraloop. ${ }^{54}$

We first measured the binding of AD2 to the tGAAAp RNA tetraloop. As shown in Figure 9, the fluorescence of tGAAAp was quenched upon addition of $0.125-10 \mu \mathrm{M}$ AD2. The fraction of RNA bound was calculated by dividing the difference between the sample and initial fluorescence by the difference between the final and initial fluorescence. We then plotted the 


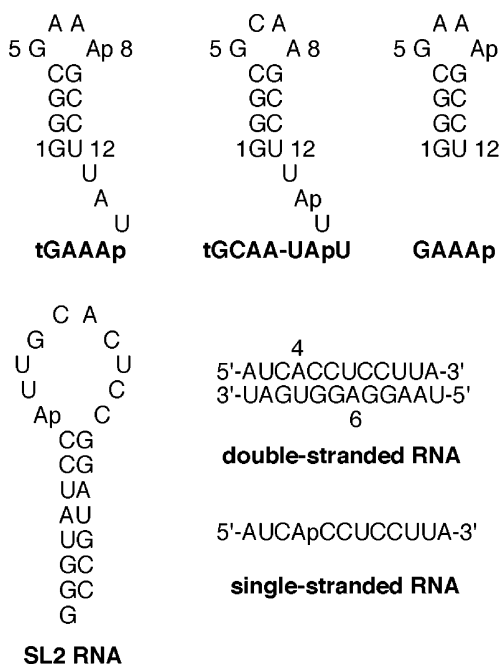

Figure 8. RNA sequences used in fluorescence experiments. Positions of labeling with 2-aminopurine are indicated with Ap. For the doublestranded sequence, some experiments were performed with RNA labeled at position 4 of the upper strand and some with RNA labeled at position 6 of the lower strand.

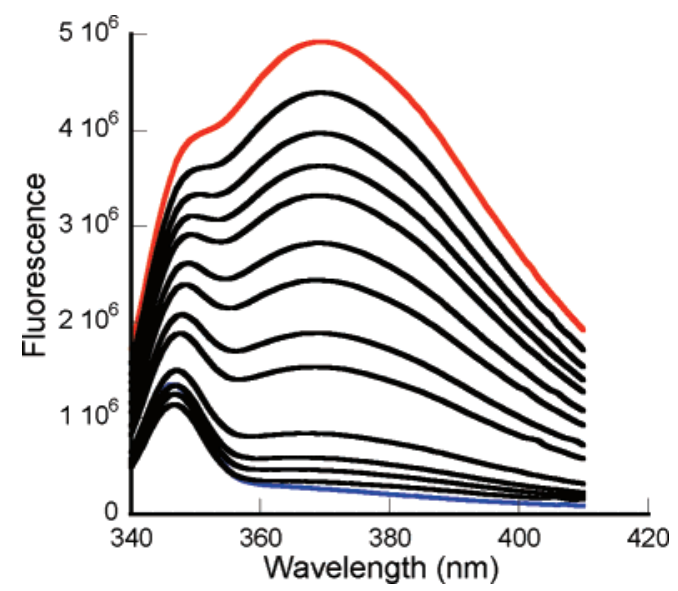

Figure 9. Fluorescence quenching of tGAAAp $(0.12 \mu \mathrm{M})$ upon addition of AD2. Experiments were performed in $10 \mathrm{mM} \mathrm{NaH}{ }_{2} \mathrm{PO}_{4}$ (pH 6.0), $0.01 \%$ Triton $\mathrm{X}-100$ at $25^{\circ} \mathrm{C}$. The concentration of $\mathrm{AD} 2$ was varied between 0.125 and $10 \mu \mathrm{M}$. The spectrum of the free tGAAAp RNA is shown in red, while that of the buffer is in blue. Samples were excited at $310 \mathrm{~nm}$, and emission was monitored from 340 to $410 \mathrm{~nm}$. Emission at $370 \mathrm{~nm}$ was used to calculate the fraction of RNA bound by AD2.

fraction of RNA bound versus the concentration of AD2, as shown in Figure 10. The data were analyzed using

$$
\frac{F-F_{0}}{F_{\mathrm{f}}-F_{0}}=\frac{K_{1}\left[\mathrm{~S}_{0}\right]+K_{1} K_{2}\left[\mathrm{~S}_{0}\right]^{2}}{1+K_{1}\left[\mathrm{~S}_{0}\right]+K_{1} K_{2}\left[\mathrm{~S}_{0}\right]^{2}}
$$

which assumes a 1:2 tGAAAp-AD2 complex. ${ }^{84} F$ is the fluorescence intensity of the sample, $F_{0}$ is the initial fluorescence intensity, $F_{\mathrm{f}}$ is the final fluorescence intensity, $\left[\mathrm{S}_{0}\right]$ is the total AD2 concentration, $K_{1}$ is the association constant of the tGAAAp-AD2 1:1 complex, and $K_{2}$ is the association constant to form the tGAAAp-(AD2) $1: 2$ complex from the $1: 1$ complex. A better fit of the data was observed assuming a 1:2, rather than a $1: 1$, binding stoichiometry. Additional binding experiments to probe the stoichiometry, which are described below, supported a 1:2 binding stoichiometry. Dissociation constants of $1.0 \pm 0.1$ and $4.0 \pm 0.6 \mu \mathrm{M}$ were obtained for the first and second binding sites, respectively (Table 2).

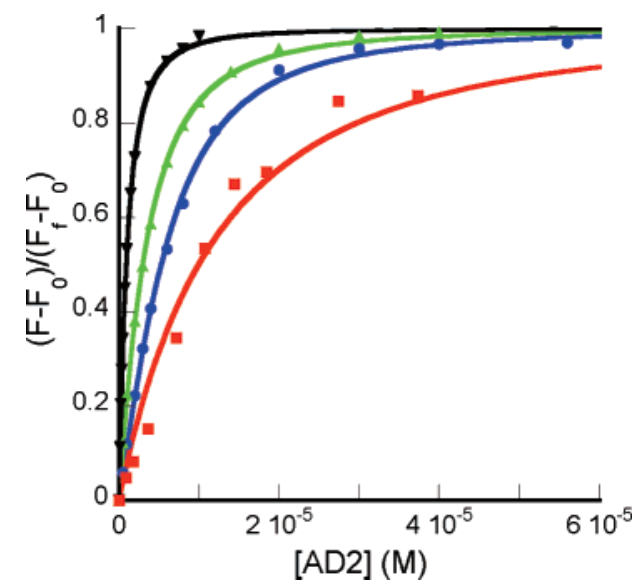

Figure 10. Representative binding curves of the complexes formed with AD2 and tGAAAp (black), GAAAp (green), single-stranded (blue), and double-stranded (red) RNAs assuming a 1:2 stoichiometry. The data were fit with eqs 1 and 2 as described in the text. Experiments were performed in $10 \mathrm{mM} \mathrm{NaH}_{2} \mathrm{PO}_{4}(\mathrm{pH} 6.0), 0.01 \%$ Triton X-100 at $25{ }^{\circ} \mathrm{C}$.

The stoichiometry of the tGAAAp-AD2 complex was measured at RNA concentrations approximately 20-fold greater than the first dissociation constant of the tGAAAp-AD2 complex. The concentration of AD2 ranged from 6.25 to 150 $\mu \mathrm{M}$, which is below the concentration at which precipitation of AD2 was observed. The fluorescence of the labeled RNA was monitored upon addition of AD2. A binding stoichiometry of two AD2 to one tetraloop RNA was indicated from three independent measurements, one of which is shown in Figure 11.

The binding affinity of AD2 for the tGCAA RNA tetraloop labeled in the 3 -overhang sequence (tGCAA-UApU) was similar to the affinity of AD2 for the tGAAAp tetraloop. The dissociation constant for the first binding site was $2.1 \pm 0.1$ $\mu \mathrm{M}$ and for the second site was $3.7 \pm 0.6 \mu \mathrm{M}$. These results suggest that the binding affinities of AD2 for the tGCAA and tGAAA tetraloops are comparable, which is not surprising given the similarity of their structures. The sensitivity of 2-aminopurine incorporated at these two different positions in the RNA to the binding of AD2 and the similarity of the dissociation constants measured with RNAs labeled in these two positions are consistent both with two binding sites and with conformational changes occurring upon binding. A conformational change in the RNA upon binding of AD2 would be expected to affect 2-aminopurine fluorescence at both positions of labeling in the RNA. Thus, an RNA labeled in the loop region would be sensitive to binding to the duplex adjacent to the 3 '-overhang and an RNA labeled in the $3^{\prime}$-overhang sequence would be sensitive to binding to the duplex adjacent to the loop.

In order to probe the contribution of the three dangling residues UAU to the binding of $\mathrm{AD} 2$, we measured the binding of AD2 to the tetraloop RNA without the 3'-overhang sequence. AD2 bound to the tetraloop with no overhang (GAAAp) at two sites with $3.3 \pm 0.4$ and $13 \pm 2 \mu \mathrm{M}$ dissociation constants. The stoichiometry of binding was determined to be two AD2 binding to one GAAAp RNA tetraloop by similar methods used to determine the stoichiometry of binding to tGAAAp (data not shown). Thus, AD2 binds with higher affinity to a helix with a 3 -overhang sequence. These results are consistent with AD2 binding to two sites on the RNA tetraloop. One site may be in the major groove proximal to the loop with a dissociation constant of approximately $4 \mu \mathrm{M}$, while the other may be in the major groove proximal to the $3^{\prime}$-overhang. The affinity of AD2 
Table 2. Dissociation Constants Determined for Complexes Formed between AD2 and Different RNA Target Sites ${ }^{a}$

\begin{tabular}{llcllcr}
\hline & tGAAAp & tGCAA-UApU & GAAAp & SL2 RNA & duplex RNA & single-stranded RNA \\
\hline$K_{\mathrm{D} 1}(\mu \mathrm{M})^{b}$ & $1.0 \pm 0.1$ & $2.1 \pm 0.1$ & $3.3 \pm 0.4$ & $0.7 \pm 0.3$ & $23 \pm 6$ & $9 \pm 2$ \\
$K_{\mathrm{D} 2}(\mu \mathrm{M})^{b}$ & $4.0 \pm 0.6$ & $3.7 \pm 0.6$ & $13 \pm 2$ & $1.7 \pm 0.7$ & $35 \pm 7$ & $9 \pm 2$ \\
\hline
\end{tabular}

${ }^{a}$ Buffer: $10 \mathrm{mM} \mathrm{NaH}_{2} \mathrm{PO}_{4}, \mathrm{pH}$ 6.06, 0.01\% Triton X-100. Equation 1 was used to fit data obtained with stem-loop RNAs. Equation 2 was used to fit data obtained with duplex and single-stranded RNA. Errors are the standard deviation of at least three independent measurements. ${ }^{b} K_{\mathrm{D} 1}$ and $K_{\mathrm{D} 2}$ are the inverse of the association constants $K_{1}$ and $K_{2}$ determined from fitting the data with eqs 1 and 2.

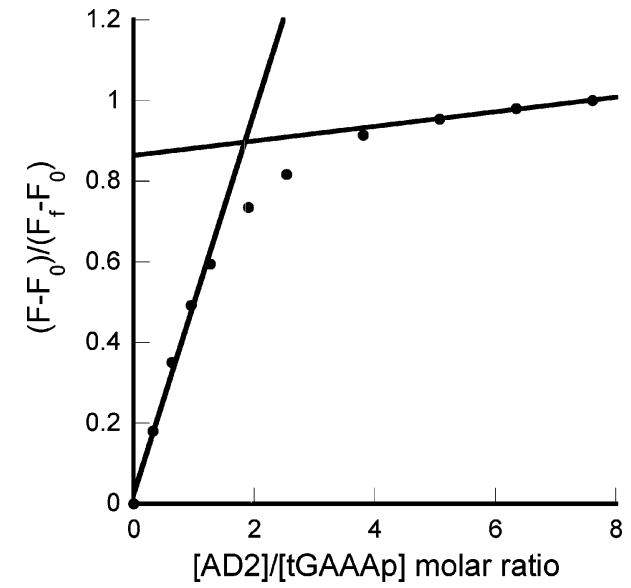

Figure 11. Plot of the fraction of RNA signal quenched versus the molar ratio of $\mathrm{AD} 2$ to tGAAAp RNA. This plot was used to determine the stoichiometry of the tGAAAp-AD2 complex. Measurements were performed with a tGAAAp concentration of $19.7 \mu \mathrm{M}$ in $10 \mathrm{mM}$ $\mathrm{NaH}_{2} \mathrm{PO}_{4}(\mathrm{pH} 6.0), 0.01 \%$ Triton $\mathrm{X}-100$ at $25^{\circ} \mathrm{C}$.

for the site proximal to the $3^{\prime}$-overhang may be destabilized by removal of the 3 -overhang, leading to an increase of the dissociation constant from $1 \mu \mathrm{M}$ for the complex formed with tGAAAp to $13 \mu \mathrm{M}$ for the complex formed with GAAAp.

Investigation of the Effect of AD2 Aggregation on Binding. Previous studies have shown that false positive hits from computational docking may arise for compounds that aggregate because the aggregates can be responsible for binding instead of the isolated molecules. ${ }^{85,86} \mathrm{We}$ have found that AD2 begins to precipitate at concentrations of approximately $200 \mu \mathrm{M}$. Thus, we probed whether AD2 aggregates were contributors to the binding of AD2 to RNA. In order to reduce the aggregation of AD2, Triton X-100 was added to the buffer. The effect of Triton $\mathrm{X}-100$ on the aggregation of small molecules has been characterized extensively by Shoiket and co-workers. ${ }^{85}$ Concentrations of $0.01 \%$ are typically sufficient to substantially diminish aggregation. If an aggregate of $\mathrm{AD} 2$ were responsible for the observed changes in 2-aminopurine fluorescence, the addition of Triton X-100 should markedly increase the measured dissociation constants. Instead, the dissociation constants of AD2-RNA complexes remained unchanged or decreased slightly in the presence of Triton X-100. For example, in the absence of detergent, dissociation constants of 3.0 and $4.0 \mu \mathrm{M}$ were determined for the complex formed between the tGAAAp tetraloop and AD2. Upon addition of $0.01 \%$ Triton X-100 to the buffer, $K_{\mathrm{D} 1}$ decreased from 3.0 to $1.0 \mu \mathrm{M}$ and $K_{\mathrm{D} 2}$ remained unchanged. Similarly, small changes in dissociation constants were observed upon addition of $0.01 \%$ Triton X-100 to the complexes formed with the tGCAA-UApU and GAAAp tetraloops. These results indicate that aggregates of $\mathrm{AD} 2$ are not responsible for the observed binding of AD2 to tetraloop RNA. All binding constants reported in Table 2 were determined using buffers containing $0.01 \%$ Triton X-100.

Binding Selectivity of AD2. In order to investigate the affinity of AD2 for different types of RNA targets, the binding of AD2 to single-stranded and double-stranded RNA and to a more flexible loop was investigated. These RNA sequences are shown in Figure 8. The sequence of the double-stranded RNA was identical to that used as a negative selection step in the computational docking. The single-stranded target was one of the strands of the double-stranded target. The flexible loop was stem loop 2 (SL2) RNA from U1 snRNA. This target was chosen because it is more flexible than the tetraloop targets, and we have previously investigated the ability of AD1 to bind to this sequence. ${ }^{50}$ All of the RNA targets were labeled with 2-aminopurine to enable binding measurements using fluorescence techniques. The double-stranded and single-stranded targets were labeled by substituting 2-aminopurine for A4 in the upper strand (5'-AUCApCCUCCUUA-3'). Double-stranded RNA was also labeled at A6 in the bottom strand ( $3^{\prime}$ UAGUGGApGGAAU-5'). Both of these positions of labeling are within the binding site used by DOCK in the computational docking described above. Binding affinities determined for duplex RNAs labeled at both positions were within experimental error. For all of the RNA targets, addition of AD2 quenched the fluorescence signal of 2-aminopurine. As with the experiments performed with the tetraloop RNA, the binding data were fit better with a $2: 1[\mathrm{AD} 2] /[\mathrm{RNA}]$ than with a 1:1 stoichiometry. Similarly to the tetraloops, the binding isotherms for AD2 with SL2 RNA were fit with eq 1 . We were unable to confirm the stoichiometry of binding to the double- and single-stranded RNAs by the method used for the RNA tetraloops because AD2 precipitated at the high concentrations required for these experiments. The following equation,

$$
\frac{F-F_{0}}{F_{\mathrm{f}}-F}=\frac{\left(K_{1}+K_{2}\right)\left[S_{0}\right]+K_{1} K_{2}\left[S_{0}\right]^{2}}{1+\left(K_{1}+K_{2}\right)\left[S_{0}\right]+K_{1} K_{2}\left[S_{0}\right]^{2}}
$$

was used to fit the data. ${ }^{84}$ This equation differs from eq 1 because it does not assume sequential binding to the two target sites. $F$ is the fluorescence intensity of the sample, $F_{0}$ is the initial fluorescence intensity, $F_{\mathrm{f}}$ is the final fluorescence intensity, $\left[\mathrm{S}_{0}\right]$ is the total $\mathrm{AD} 2$ concentration, $K_{1}$ is the association constant of RNA-AD2 at one binding site, and $K_{2}$ is the association constant of RNA-AD2 at the other binding site.

The data, shown in Figure 10 and Table 2, indicate that AD2 binds with higher affinity to hairpin structures than to singleand double-stranded RNA target sites. Binding of AD2 to singlestranded RNA is 9-fold weaker than to the tGAAAp RNA for the first binding site, while the binding of AD2 to the doublestranded RNA is more than 20-fold weaker than to the tGAAAp RNA for the first binding site. In contrast, the affinity of AD2 for the SL2 of U1 snRNA is similar to or even slightly greater than for the tetraloop RNA. These results suggest that AD2 prefers to bind to structures with a double-stranded to singlestranded junction, such as those found in hairpins, perhaps because they have a wider major groove in positions proximal to the loop than is found in regular A-form duplexes.

\section{Conclusion}

In conclusion, we have used computational docking to identify molecules that bind RNA tetraloops. Incorporation of a negative 
selection step in the computational docking to reduce the number of molecules that would bind double-stranded RNA allowed us to identify compounds that bind with significant selectivity to hairpin over double-stranded RNA. The similar binding affinity of AD2 for tetraloop and SL2 RNAs and the destabilization of the complex upon elimination of the 3'-overhang of the stem suggest that AD2 may bind generally to junctions between single- and double-stranded RNA.

We have previously found that a related molecule, AD1, binds to tetraloop and duplex RNAs with similar affinity. AD1 and AD2 share the same acridine core but have different side chains. The flexible side chain of AD1 may allow it to interact with multiple RNA binding sites. We and others have shown that AD1 binds to stem-loop 2 of U1 snRNA, ${ }^{50}$ HIV-1 TAR RNA, ${ }^{56,87}$ tetraloop RNA, ${ }^{54}$ and duplex RNA. ${ }^{57}$ The rigidity of the side chain of $\mathrm{AD} 2$ relative to that of $\mathrm{AD} 1$ may be responsible for the increased selectivity of RNA binding. It is interesting to note that the binding affinities of AD1 and AD2 for tetraloop RNA are similar, while the binding affinity of AD2 for duplex RNA is considerably lower than that of AD1 for duplex RNA. Thus, the increased selectivity of AD2 compared to AD1 was achieved by destabilizing the complex formed with duplex RNA rather than stabilizing that formed with tetraloop RNA.

This work suggests that the acridine derivatives described here could form the basis for the development of molecules that specifically recognize different hairpin and overhang structures. In fact, related acridine derivatives have recently been found that bind with 70-fold selectivity for quadruplex DNA over double-stranded DNA. ${ }^{88}$ Improvement of selectivity for tetraloop RNA over other stem-loop and overhang structures will require modification of $\mathrm{AD} 2$ with groups that specifically recognize the sequence or structural features of the loop nucleotides.

\section{Experimental Section}

Materials. RNA sequences were obtained from Dharmacon Research Inc. (Lafayette, CO). RNAs were first deprotected following the protocol provided by Dharmacon and then purified by $20 \%$ denaturing polyacrylamide gel electrophoresis. RNA bands were visualized by UV and excised from the gel. RNAs were extracted from the gel slices either by electroelution using an Elutrap from Schleicher \& Schuell or by TE (10 mM Tris, 1 mM EDTA, $\mathrm{pH}$ 7.5) extraction. The RNAs were then desalted by three to four ethanol precipitations and extensive dialysis against water. For fluorescence experiments, the purity and identity of the RNA were confirmed by analytic PAGE and MALDI-MS. For NMR experiments performed in $\mathrm{D}_{2} \mathrm{O}$, purified RNA samples were dissolved in $600 \mu \mathrm{L}$ of $10 \mathrm{mM} \mathrm{NaH}_{2} \mathrm{PO}_{4}(\mathrm{pH} 6$ ), dried under reduced pressure, and dissolved in $600 \mu \mathrm{L}$ of $99.9 \% \mathrm{D}_{2} \mathrm{O}$. The RNA samples were then annealed by heating to $368 \mathrm{~K}$ for $5 \mathrm{~min}$ followed by cooling on ice for 5 min immediately before the NMR experiments.

All compounds under investigation were obtained from the Drug Synthesis and Chemistry Branch, Developmental Therapeutics Program, Division of Cancer Treatment and Diagnosis, National Cancer Institute (Bethesda, MD). The NCI diversity set is a free database from the National Cancer Institute. The NCI code for AD2 is NSC 130813-V.

Computational work was performed on a Silicon Graphics Origin 200 with a CPU of $4 \times$ R10000 @ $180 \mathrm{MHz}, 512 \mathrm{MB}$ RAM, and an Irix 6.5.5 operating system. The DOCK 5.1.1 program was provided free of charge by UCSF,, 990 and AutoDock 3.0 was obtained free of charge from the Molecular Graphics Laboratory of the Scripps Research Institute. ${ }^{74}$ Insight II from Accelrys was used to add atomic partial charges to the RNA and small molecules.

Computational Screening with DOCK and AutoDock. The 1990 compounds in the NCI diversity set were screened for binding to RNA tetraloops. Compounds were grouped in 20 groups with
100 compounds in each group. We first removed the compounds whose molecular weight was larger than 500 . Hydrogens and the partial atomic charges were added to the compounds using the Insight II CFF91 force field. Some inorganic compounds were removed from the database because they were not charged properly.

A solution structure of a GAAA tetraloop RNA was selected as the RNA target for docking and experimental studies. ${ }^{28}$ Its atomic coordinates (1ZIF) were obtained from the Brookhaven Protein Databank (PDB). RNA was also charged by the CFF91 force field. The binding site was generated using the SPHGEN ${ }^{90}$ module in DOCK, and the docking site was chosen to be the first cluster of 38 spheres in the major groove of the tetraloop RNA. Flexible ligand docking was enabled with sampling of 1000 conformations. Compounds were ranked, and those in the top 50\% were subject to further docking to the duplex RNA.

A crystal structure of r(UAAGGAGGUGAU) - r(AUCACCUCCUUA) was selected as a model structure for an A-form RNA duplex. ${ }^{83}$ The PDB code for this duplex RNA is 1SDR. Docking sites were generated as previously described by Kuntz and co-workers. ${ }^{64}$ The top scoring $50 \%$ of the NCI compounds from the previous step were docked to the RNA duplex, and the docking energies of each compound binding to tetraloop and double-stranded RNA were compared. The compounds that were predicted to bind with higher affinity to tetraloop RNA than duplex RNA were selected for the next round of docking. These 350 compounds were docked to the tetraloop RNA using AutoDock in order to obtain a more accurate estimate of binding energy. The default parameters were used for docking. Ligand pdbq files were generated by Scripps Research Institute and are freely available from http://www. scripps.edu/mb/olson/doc/autodock/. ${ }^{74,91}$

Fluorescence Experiments. Fluorescence experiments were carried out on a FluoroMax-3 Spex spectrofluorometer from Jobin Yvon Inc. The change of the intensity of the 2-aminopurine fluorescence upon addition of AD2 was monitored. RNA was excited at $310 \mathrm{~nm}$, and emission scans were acquired from 340 to $410 \mathrm{~nm}$ with excitation and emission slits less than or equal to 5 $\mathrm{nm}$. An average of six scans was obtained, and the dark count correction was enabled. The fraction of RNA bound was calculated using the emission at $370 \mathrm{~nm}$ by dividing the difference between the observed fluorescence $F$ and the initial fluorescence $F_{0}$ by the difference between final fluorescence $F_{\mathrm{f}}$ and initial fluorescence $\mathrm{F}_{0}$ as shown in eq 1 . The plots of fraction bound vs total concentration of AD2 were fit by eq 1 assuming a 1:2 stoichiometry and sequential binding for stem loop target sites. ${ }^{84}$

The plots of fraction bound vs total concentration of AD2 were fit by eq 2 assuming a one to two stoichiometry but not sequential binding for single- and double-stranded target sites.

Both fitting equations assume that the fluorescence signal change is similar for binding to either of the two binding sites. For both sets of plots, concentrations of free AD2 were used instead of total concentration of AD2 when the concentration of RNA was within a factor of 5 of the $K_{\mathrm{D}}$ so that [AD2] could not be approximated as $[\mathrm{AD} 2]_{\text {total }}$.

NMR Experiments. The NMR experiments were carried out using a Varian $400 \mathrm{MHz}$ UnityPlus spectrometer or Varian 500 $\mathrm{MHz}$ spectrometer. The $1 \mathrm{D}{ }^{1} \mathrm{H}$ NMR titration experiments were performed with a sweep width of $6000 \mathrm{~Hz}, 128$ or 256 transients, a $2 \mathrm{~s}$ delay time for the experiments in $\mathrm{H}_{2} \mathrm{O}$, and a $5 \mathrm{~s}$ delay time for the experiments in $\mathrm{D}_{2} \mathrm{O}$. Chemical shifts of $\mathrm{AD} 2$ were broadened upon binding at $298 \mathrm{~K}$ because of intermediate exchange. Therefore, some experiments were performed at $313 \mathrm{~K}$ in order to obtain sharper peaks for AD2. A 2D NOE spectrum of tGCAA was acquired to make chemical shift assignments. The 2D NOESY data were collected with a mixing time of $400 \mathrm{~ms}, 5 \mathrm{~s}$ of delay time, a sweep width of $4000 \mathrm{~Hz}$ in both dimensions, 32 transients, and 356 increments.

CD Spectroscopy. CD experiments were carried out using a JASCO J-810 spectropolarimeter. Increasing concentrations of AD2 (3-70 $\mu \mathrm{M}$ ) were added to $12 \mu \mathrm{M}$ tGCAA in $10 \mathrm{mM} \mathrm{NaH}{ }_{2} \mathrm{PO}_{4}$, $\mathrm{pH}$ 6. At least three spectral scans were accumulated over a 
wavelength range from 200 to $320 \mathrm{~nm}$ and a temperature of $25^{\circ} \mathrm{C}$ in a $0.2 \mathrm{~cm}$ path-length cell at a scanning rate of $20 \mathrm{~nm} / \mathrm{min}$.

Acknowledgment. Compounds, including AD2, that were experimentally tested for binding to tetraloop RNA were provided by the National Cancer Institute. We thank Dr. Irwin Kuntz, Terry Downing, and Demetri Moustakas of UCSF for assistance with DOCK. We are grateful to Dr. Arthur Pardi of the University of Colorado for sending us the NMR chemical shift assignments of the RNA tetraloops. We thank Professor Philip Bolton and Dr. Iulian Rujan for assistance with the NMR experiments. This research was supported by a Mellon New Initiative grant from Wesleyan University.

\section{References}

(1) Bloomfield, V. A.; Crothers, D. M.; Tinoco, I. Nucleic Acids: Structures, Properties, and Functions; University Science Books: Sausalito, CA, 2000.

(2) Chow, C.; Bogdan, F. A structural basis for RNA-ligand interactions. Chem. Rev. 1997, 97, 1489-1513.

(3) Sutcliffe, J. A. Improving on nature: antibiotics that target the ribosome. Curr. Opin. Microbiol. 2005, 8, 534-542.

(4) Wilson, W. D.; Li, K. Targeting RNA with small molecules. Curr. Med. Chem. 2000, 7, 73-98.

(5) Krebs, A.; Ludwig, V.; Boden, O.; Göbel, M. W. Targeting the HIV trans-activation responsive region-approaches towards RNA-binding drugs. ChemBioChem 2003, 4, 972-978.

(6) Hermann, T. Drugs targeting the ribosome. Curr. Opin. Struct. Biol. $\mathbf{2 0 0 5}, 15,355-366$.

(7) Foloppe, N.; Matassova, N.; Faboul-ela, F. Towards the discovery of drug-like RNA ligands? Drug Discovery Today 2006, 11, 10191027.

(8) Moore, P. B. Structural motifs in RNA. Annu. Rev. Biochem. 1999, 67, 287-300.

(9) Woese, C. R.; Gutell, R.; Gupta, R.; Noller, H. F. Detailed analysis of the higher-order structure of 16s-like ribosomal ribonucleic-acids. Microbiol. Rev. 1983, 47, 621-669.

(10) Woese, C. R.; Winker, S.; Gutell, R. R. Architecture of ribosomal RNA: constraints on the sequence of "tetra-loops". Proc. Natl. Acad. Sci. U.S.A. 1990, 87, 8467-8471.

(11) Varani, G. Exceptionally stable nucleic acid hairpins. Annu. Rev. Biophys. Biomol. Struct. 1995, 24, 379-404.

(12) Klosterman, P. S.; Hendrix, D. K.; Tamura, M.; Holbrook, S. R.; Brenner, S. E. Three dimensional motifs from the SCOR, structural classification of RNA database: extruded strands, base triples, tetraloops and U-turns. Nucleic Acids Res. 2004, 32, 2342-2352.

(13) Krummel, D. A. P.; Altman, S. Verification of phylogenetic predictions in vivo and the importance of the tetraloop motif in a catalytic RNA. Proc. Natl. Acad. Sci. U.S.A. 1999, 96, 11200-11205.

(14) Ikawa, Y.; Naito, D.; Aono, N.; Shiraishi, H.; Inoue, T. A conserved motif in group IC3 introns is a new class of GNRA receptor. Nucleic Acids Res. 1999, 27, 1859-1865.

(15) Costa, M.; Deme, E.; Jacquier, A.; Michel, F. Multiple Tertiary interactions involving domain II of group II self-splicing introns. $J$. Mol. Biol. 1997, 267, 520-536.

(16) Costa, M.; Michel, F. Frequent use of the same tertiary motif by self-folding RNAs. EMBO J. 1995, 14, 1276-1285.

(17) Bélanger, F.; Gagnon, M. G.; Steinberg, S. V.; Cunningham, P. R.; Brakier-Gingras, L. Study of the functional interaction of the 900 tetraloop of 16S ribosomal RNA with helix 24 within the bacterial ribosome. J. Mol. Biol. 2004, 338, 683-693.

(18) Hedenstierna, K. O. F.; Siefert, J. L.; Fox, G. E.; Murgola, E. J. Co-conservation of rRNA tetraloop sequences and helix length suggests involvement of the tetraloops in higher-order interactions. Biochimie 2000, 82, 221-227.

(19) Fernández-miragall, O.; Martínez-salas, E. Structural organization of a viral IRES depends on the integrity of the GNRA motif. RNA 2003, 9, 1333-1344.

(20) Psaridi, L.; Georgopoulou, U.; Varaklioti, A.; Mavromara, P. Mutational analysis of a conserved tetraloop in the $5^{\prime}$ untranslated region of hepatitis $\mathrm{C}$ virus identifies a novel RNA element essential for the internal ribosome entry site function. FEBS Lett. 1999, 453, 49-53.

(21) Fernández-miragall, O.; Ramos, R.; Ramajo, J.; Martínez-salas, E. Evidence of reciprocal tertiary interactions between conserved motifs involved in organizing RNA structure essential for internal initiation of translation. RNA 2006, 12, 223-234.

(22) Zwieb, C. Conformity of RNAs that interact with tetranucleotide loop binding proteins. Nucleic Acids Res. 1992, 20, 4397-4400.
(23) Jagath, J. R.; Matassova, N. B.; De Leeuw, E.; Warnecke, J. M.; Lentzen, G.; Rodnina, M. V.; Luirink, J.; Wintermeyer, W. Important role of the tetraloop region of 4.5S RNA in SRP binding to its receptor Fts Y. RNA 2001, 7, 293-301.

(24) Glück, A.; Endo, Y.; Wool, I. G. Ribosomal RNA identity elements for ricin A-chain recognition and catalysis. J. Mol. Biol. 1992, 226, 411-424.

(25) Zeffman, A.; Hassard, S.; Varani, G.; Lever, A. The major HIV-1 packaging signal is an extended bulged stem loop whose structure is altered on interaction with the Gag polyprotein. J. Mol. Biol. 2000, 297, 877-893.

(26) Shu, F. Y.; Spanggord, R. J.; Doudna, J. A. SRP RNA provides the physiologically essential GRPase activation function in cotranslational protein targeting. RNA 2007, 13, 240-250.

(27) Heus, H. A.; Pardi, A. Structural features that give rise to the unusual stability of RNA hairpins containing GNRA loops. Science 1991, 253, 191-194.

(28) Jucker, F. M.; Heus, H. A.; Yip, P. F.; Moors, E. H. M.; Pardi, A. A network of heterogeneous hydrogen bonds in GNRA tetraloops. J. Mol. Biol. 1996, 264, 968-980.

(29) Varani, B.; Cheong, C.; Tinoco, I. Structure of an unusually stable RNA hairpin. Biochemistry 1991, 30, 3280-3289.

(30) Rüdisser, S.; Tinoco, I. Solution structure of cobalt(III) hexammine complexed to the GAAA tetraloop, and metal-ion binding to $\mathrm{G} \cdot \mathrm{A}$ mismatches. J. Mol. Biol. 2000, 295, 1211-1223.

(31) Davis, J. H.; Tonelli, M.; Scott, L. G.; Jaeger, L.; Williamson, J. R.; Butcher, S. E. RNA Helical packing in solution: NMR structure of a $30 \mathrm{kDa}$ GAAA tetraloop-receptor complex. J. Mol. Biol. 2005, $351,371-382$

(32) Pley, H. W.; Flaherty, K. M.; Mckay, D. B. Model for an RNA tertiary interaction from the structure of an intermolecular complex between a GAAA tetraloop and an RNA helix. Nature 1994, 372, $111-113$

(33) Cate, J. H.; Gooding, A. R.; Podell, E.; Zhou, K.; Golden, B. L.; Kundrot, C. E.; Cech, T. R.; Doudna, J. A. Crystal structure of a group I ribozyme domain: principles of RNA packing. Science 1996, $273,1678-1685$

(34) Correll, C. C.; Swinger, K. Common and distinctive features of GNRA tetraloops based on a GUAA tetraloop structure at $1.4 \AA$ resolution. RNA 2003, 9, 355-363.

(35) Correll, C. C.; Wool, I. G.; Munishkin, A. The two faces of the Escherichia coli 23 S rRNA sarcin/ricin domain: the structure at 1.11 A resolution. J. Mol. Biol. 1999, 292, 275-287.

(36) Perbandt, M.; Nolte, A.; Lorenz, S.; Bald, R.; Betzel, C.; Erdmann, V. A. Crystal structure of domain E of Thermus flavus 5 S rRNA: a helical RNA structure including a hairpin loop. FEBS Lett. 1998, 429, 211-215.

(37) Scott, W. G.; Finch, J. T.; Klug, A. The crystal structure of an allRNA hammerhead ribozyme: a proposed mechanism for RNA catalytic cleavage. Cell 1995, 81, 991-1002.

(38) SantaLucia, J.; Kierzek, R.; Turner, D. H. Context dependence of hydrogen bond free energy revealed by substitutions in an RNA hairpin. Science 1992, 256, 217-219.

(39) Wörner, K.; Strube, T.; Engels, J. W. Synthesis and stability of GNRA-loop analogs. Helv. Chim. Acta 1999, 82, 2094-2104.

(40) Antao, V. P.; Lai, S. Y.; Tinoco, I. A thermodynamic study of unusually stable RNA and DNA hairpins. Nucleic Acids Res. 1991, 19, 5901-5905.

(41) Leuilliot, N.; Baumruk, V.; Abdelkafi, M.; Turpin, P.-Y.; Namane, A.; Gouyette, C.; Huynh-Dinh, T.; Ghomi, M. Unusual nucleotide conformations in GNRA and UNCG type tetraloop hairpins: evidence from Raman markers assignments. Nucleic Acids Res. 1999, 27, 1398-1404.

(42) Menger, M.; Eckstein, F.; Porschke, D. Dynamics of the RNA hairpin GNRA tetraloop. Biochemistry 2000, 39, 4500-4507.

(43) Ma, H.; Proctor, D. J.; Kierzek, E.; Kierzek, R.; Bevilacqua, P. C.; Gruebele, M. Exploring the energy landscape of a small RNA hairpin J. Am. Chem. Soc. 2006, 128, 1523-1530.

(44) Hodak, J. H.; Fiore, J. L.; Nesbitt, D. J.; Downey, C. D.; Pardi, A. Docking kinetics and equilibrium of a GAAA tetraloop-receptor motif probed by single-molecule FRET. Proc. Natl. Acad. Sci. U.S.A. 2005, 102, 10505-10510.

(45) Sorin, E. J.; Engelhardt, M. A.; Herschlag, D.; Pande, V. S. RNA Simulations: probing hairpin unfolding and the dynamics of a GNRA tetraloop. J. Mol. Biol. 2002, 317, 493-506.

(46) Sarzynska, J.; Nilsson, L.; Kulinski, T. Effects of base substitutions in an RNA hairpin from molecular dynamics and free energy simulations. Biophys. J. 2003, 85, 3445-3459.

(47) Li, W.; Ma, B.; Shapiro, B. A. Molecular dynamics simulations of the denaturation and refolding of an RNA tetraloop. J. Biomol. Struct. Dyn. 2001, 19, 381-396.

(48) Thomas, J. R.; Liu, X.; Hergenrother, P. J., Size-specific ligands for RNA hairpin loops. J. Am. Chem. Soc. 2005, 127, 12434-12435. 
(49) Liu, X.; Thomas, J. R.; Hergenrother, P. J. Deoxystreptamine dimers bind to RNA hairpin loops. J. Am. Chem. Soc. 2004, 126, 91969197.

(50) Gayle, A. Y.; Baranger, A. M. Inhibition of the U1A-RNA complex by an aminoacridine derivative. Bioorg. Med. Chem. Lett. 2002, 12 , $2839-2842$.

(51) Thomas, J. R.; Liu, X.; Hergenrother, P. J. Biochemical and thermodynamic characterization of compounds that bind to RNA hairpin loops: toward an understanding of selectivity. Biochemistry 2006, 45, 10928-10938.

(52) Verhelst, S. H. L.; Michiels, P. J. A.; van der Marel, G. A.; van Boeckel, C. A. A.; van Boom, J. H. Surface plasmon resonance evaluation of various aminoglycoside-RNA hairpin interactions reveals low degree of selectivity. ChemBioChem 2004, 5, 937-942.

(53) Kaiser, M.; Sainlos, M.; Lehn, J.-M.; Bombard, S.; Teulade-Fichou, M.-P. Aminoglycoside-quinacridine conjugates: towards recognition of the P6.1 element of telomerase RNA. ChemBioChem 2006, 7, 321-329.

(54) Yan, Z.; Baranger, A. M. Binding of an aminoacridine derivative to a GAAA RNA tetraloop. Bioorg. Med. Chem. Lett. 2004, 14, 58895893.

(55) Gelus, N.; Hamy, F.; Bailly, C. Molecular basis of HIV-1 TAR RNA specific recognition by an acridine tat-antagonist. Bioorg. Med. Chem. 1999, 7, 1075-1079.

(56) Hamy, F.; Brondani, V.; Flörsheimer, A.; Stark, W.; Blommers, M. J. J.; Klimkait, T. A new class of HIV-1 Tat antagonist acting through Tat-TAR inhibition. Biochemistry 1998, 37, 5086-5095.

(57) Yan, Z.; Baranger, A. M. Unpublished data.

(58) Leach, A. R.; Shoichet, B. K.; Peishoff, C. E. Prediction of proteinligand interactions. Docking and scoring: successes and gaps. J. Med. Chem. 2006, 49, 5851-5855.

(59) Ghosh, S.; Nie, A.; An, J.; Huang, Z. Structure-based virtual screening of chemical libraries for drug discovery. Curr. Opin. Chem. Biol. 2006, 10, 194-202.

(60) Detering, C.; Varani, G. Validation of automated docking programs for docking and database screening against RNA drug targets. J. Med. Chem. 2004, 47, 4188-4201.

(61) Foloppe, N.; Chen, I. J.; Davis, B.; Hold, A.; Morley, D.; Howes, R A structure-based strategy to identify new molecular scaffolds targeting the bacterial ribosomal A-site. Bioorg. Med. Chem. 2004, 12, 935-947.

(62) Morley, S. D.; Afshar, M. Validation of an empirical RNA-ligand scoring function for fast flexible docking using RiboDock. $J$. Comput.-Aided Mol. Des. 2004, 18, 189-208.

(63) Chen, Q.; Kuntz, I. D.; Shafer, R. H. Structure-Based Design of Nucleic Acid Binding Drugs. In Structure, Motion, Interaction and Expression of Biological Macromolecules; Adenine Press: New York, 1997; pp 227-231.

(64) Chen, Q.; Shafer, R. H.; Kuntz, I. D., Structure-based discovery of ligands targeted to the RNA double helix. Biochemistry 1997, 36, 11402-11407.

(65) Filikov, A. V.; Mohan, V.; Vickers, T. A.; Griffey, R. H.; Cook, P D.; Abagyan, R. A.; James, T. L. Identification of ligands for RNA targets via structure-based virtual screening: HIV-1 TAR. J. Comput.Aided Mol. Des. 2000, 14, 593-610.

(66) Haddad, J.; Kotra, L. P.; Liano-Sotelo, B.; Kim, C.; Azucena, E. F.; Liu, M.; Vakulenko, S. B.; Chow, C. S.; Mobashery, S. Design of novel antibiotics that bind to the ribosomal acyltransfer site. J. Am. Chem. Soc. 2002, 124, 3229-3237.

(67) Lind, K. E.; Du, Z.; Fujinaga, K.; Peterlin, B. M.; James, T. L. Structure-based computational database screening, in vitro assay, and NMR assessment of compounds that target TAR RNA. Chem. Biol. 2002, 9, 185-193.

(68) Zhang, X.; Wang, X.; Liu, C. Molecular docking and 3D-QSAR study of pyranmycin derivatives against $16 \mathrm{~S}$ rRNA A site. J. Mol. Struct.: THEOCHEM 2005, 730, 85-94.

(69) Barbault, F.; Zhang, L.; Zhang, L.; Fan, B. T. Parametrization of a specific free energy function for automated docking against RNA targets using neural networks. Chem. Intell. Lab. Syst. 2006, 82, 269275 .

(70) Evans, D. A.; Neidle, S. Virtual screening of DNA minor groove binders. J. Med. Chem. 2006, 49, 4232-4238.

(71) Österberg, F.; Morris, G. M.; Sanner, M. F.; Olson, A. J.; Goodsell, D. S. Automated docking to multiple target structures: incorporation of protein mobility and structural water heterogeneity in AutoDock. Proteins 2002, 46, 34-40.

(72) Moitessier, N.; Westhof, E.; Hanessian, S. Docking of aminoglycosides to hydrated and flexible RNA. J. Med. Chem. 2006, 49, 10231033 .
(73) Ewing, T. J. A.; Makino, S.; Skillman, A. G.; Kuntz, I. D. DOCK 4.0: search strategies for automated molecular docking of flexible molecule databases. J. Comput.-Aided Mol. Des. 2001, 15, 411428.

(74) Morris, G. M.; Goodsell, D. S.; Halliday, R. S.; Huey, R.; Hart, W. E.; Belew, R. K.; Olson, A. J. Automated docking using a Lamarckian genetic algorithm and an empirical binding free energy function. $J$. Comput. Chem. 1998, 19, 1639-1662.

(75) Warren, G. L.; Andrews, C. W.; Capelli, A.-M.; Clarke, B.; LaLonde, J.; Lambert, M. H.; Lindvall, M.; Nevins, N.; Semus, S. F.; Senger, S.; Tedesco, G.; Wall, I. D.; Woolven, J. M.; Peishoff, C. E.; Head, M. S. A critical assessment of docking programs and scoring functions. J. Med. Chem. 2006, 49, 5912-5931.

(76) Yang, Y.; Kochayan, M.; Burgstaller, P.; Westhof, E.; Famulok, M. Structural basis of ligand discrimination by two related RNA aptamers resolved by NMR spectroscopy. Science 1996, 272, 1343-1347.

(77) Nix, J.; Sussman, D.; Wilson, C. The 1.3 angstrom crystal structure of a biotin-binding pseudoknot and the basis for RNA molecular recognition. J. Mol. Biol. 2000, 296, 1235-1244.

(78) Zimmermann, G. R.; Jenison, R. D.; Wick, C. L.; Simorre, J.-P.; Pardi, A. Interlocking structural motifs mediate molecular discrimination by a theophylline-binding RNA. Nat. Struct. Biol. 1997, 4, 644649.

(79) Lind, K. E.; Du, Z.; Fujinaga, K.; Peterlin, B. M.; James, T. L. Structure-based computational database screening, in vitro assay, and NMR assessment of compounds that target TAR RNA. Chem. Biol. 2002, 9, 185-193.

(80) Heus, H. A.; Pardi, A. Structural features that give rise to the unusual stability of RNA hairpins containing GNRA loops. Science 1991 253, 191-193.

(81) Jucker, F. M.; Heus, H. A.; Yip, P. F.; Moors, E. H. M.; Pardi, A. A Network of heterogeneous hydrogen bonds in GNRA tetraloops. J. Mol. Biol. 1996, 264, 968-980.

(82) Rudisser, S.; Tinoco, I., Jr. Solution structure of cobalt(III) hexamine complexed to the GAAA tetraloop, and metal-ion binding to GA mismatches. J. Mol. Biol. 2000, 295, 1211-1223.

(83) Schindelin, H.; Zhang, M.; Bald, R.; Fürste, J.-P.; Erdmann, V. A. Heinemann, U. Crystal structure of an RNA dodecamer containing the Escherichia coli Shine-Dalgarno sequence. J. Mol. Biol. 1995, 249, 595-603.

(84) Eftink, M. R. Fluorescence methods for studying equilibirum macromolecule-ligand interactions. Methods Enzymol. 1997, 278, 221-257.

(85) McGovern, S. L.; Helfand, B. T.; Feng, B.; Shoichet, B. K. A specific mechanism of nonspecific inhibition. J. Med. Chem. 2003, 46, 42654272

(86) Seidler, J.; McGovern, S. L.; Doman, T. N.; Shoichet, B. K Identification and prediction of promiscuous aggregating inhibitors among known drugs. J. Med. Chem. 2003, 46, 4477-4486.

(87) Hamy, F.; Felder, E. R.; Heizmann, G.; Lazdins, J.; Aboul-Ela, F.; Varani, G.; Karn, J.; Klimkait, T. An inhibitor of the Tat/TAR RNA interaction that effectively suppresses HIV-1 replication. Proc. Natl. Acad. Sci. U.S.A. 1997, 94, 3548-3553.

(88) Moore, M. J. B.; Schultes, C. M.; Cuesta, J.; Cuenca, F.; Gunaratnam, M.; Tanious, F. A.; Wilson, W. D.; Neidle, S. Trisubstituted acridines as G-quadruplex telomere targeting agents. Effects of extensions of the 3,6- and 9-side chains on quadruplex binding, telomerase activity, and cell proliferation. J. Med. Chem. 2006, 49, 582-599.

(89) Kuntz, I. D. Structure-based strategies for drug design and discovery. Science 1992, 257, 1078-1082.

(90) Kuntz, I. D.; Blaney, J. M.; Oatley, S. J.; Langridge, R.; Ferrin, T E. A geometric approach to macromolecule-ligand interactions. $J$. Mol. Biol. 1982, 161, 269-288.

(91) Lindstorn, W. H.; Morris, G. M.; Huey, R. H.; Sanner, M. F.; Olson, A. J. The NCI Diversity Set for AutoDock. http://www.scrips.edu/ pub/olson-web/doc/autodock/, 2003.

(92) Famulok, M. Molecular recognition of amino acids by RNA-aptamers an L-citrulline binding RNA motif and its evolution into an L-arginine binder. J. Am. Chem. Soc. 1994, 116, 1698-1706.

(93) Wilson, C.; Nix, J.; Szostak, J. Functional requirements for specific ligand recognition by a biotin-binding RNA pseudoknot. Biochemistry 1998, 37, 14410-14419.

(94) Jenison, R. D.; Gill, S. C.; Pardi, A.; Polisky, B. High-resolution molecular discrimination by RNA. Science 1994, 263, 1425-1429.

\section{JM070305P}

